Research report

\title{
Pain and depression in older people: Comorbidity and patterns of help seeking
}

\author{
A. Bonnewyn ${ }^{\text {a,* }}$, C. Katona ${ }^{\text {b }}$, R. Bruffaerts ${ }^{\text {a }}$, J.M. Haro ${ }^{\text {c }}$, R. de Graaf ${ }^{\mathrm{d}}$, J. Alonso ${ }^{\mathrm{e}}$, K. Demyttenaere ${ }^{\mathrm{a}}$ \\ a University Psychiatric Center KuLeuven, Campus Gasthuisberg, Leuven, Belgium

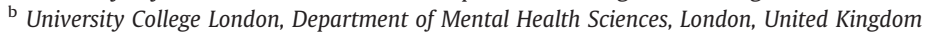 \\ ' Fundació Sant Joan de Deu, Barcelona, Spain \\ ${ }^{\mathrm{d}}$ Netherlands Institute of Mental Health and Addiction, Utrecht, The Netherlands \\ e Health Services Research Unit, Institut Municipal d'Investigacio Medica (IMIM-IMAS), Barcelona, Spain
}

\section{A R T I C L E I N F O}

\section{Article history:}

Received 17 October 2008

Received in revised form 17 December 2008

Accepted 14 January 2009

Available online 13 February 2009

\section{Keywords:}

Elderly

Pain

Major depressive disorder

Comorbidity

Help-seeking

\begin{abstract}
A B S T R A C T
Background: Chronic pain and mood disorders are common in older people. Their relationship however remains unclear. Only a few studies have investigated the role of pain in mental health service use and received psychopharmacological treatment.

Methods: Cross-sectional study of the 65+ subsample from the European Study on the Epidemiology of Mental Disorders (ESEMeD). 4401 non-institutionalized individuals were interviewed using the third version of the Composite International Diagnostic Interview (CIDI-3.0). Results: Painful physical symptoms (PPS) were more likely in people with a 12-month major depressive episode (MDE) than in those without $(\mathrm{OR}=2.0)$. Help seeking for emotional problems was uncommon, but PPS were a significant predictor of help-seeking $(\mathrm{OR}=1.7)$. Respondents with MDE more frequently used benzodiazepines than antidepressants. The presence of PPS in respondents without depression resulted in a significant increase in the use of psychotropic medication.

Conclusions: PPS were strongly and independently associated with major depressive disorder. Their presence had an influence on help seeking behavior and use of psychotropic medication. Limitations: The cross-sectional nature of this study does not allow determination of direction of causality.
\end{abstract}

(c) 2009 Elsevier B.V. All rights reserved.

\section{Introduction}

In older people, the presence of major depression is not uncommon. Population studies have indicated prevalence rates ranging from 1-5\% (Hybels and Blazer, 2003). The prevalence of subsyndromal, clinical depressive symptoms appears to be even higher with rates ranging from 3-26\% (Hybels and Blazer, 2003). The presence of pain is in the elderly a common state, with an estimated prevalence ranging from 25-88\% (Helme and Gibson, 2001). Consequently, comorbidity of pain and depression is high, although, in contrast to the younger adult population, their interaction has received relatively little attention. Prospective studies

\footnotetext{
* Corresponding author.

E-mail address: anke.bonnewyn@uz.kuleuven.ac.be (A. Bonnewyn).
}

have found an association between pain and depression in two directions. Chronic pain conditions increase the risk for depressive symptoms (consequence hypothesis) (Chou and Chi, 2005). Conversely, the presence of depressive symptoms increases the risk of onset of pain over time (antecedent hypothesis) (Reid et al., 2003). Consequently, this robust depression-pain dyad is considered as having an intimate and probably reciprocal character (Meyer et al., 2007; Chou, 2007), although the pathogenic pathways leading to depression and/or pain remain unclear. Moreover, the association is considered to be mediated by other variables such as gender (a stronger association in women, Geerlings et al., 2002; Onder et al., 2005) and disability (Williamson and Schultz, 1992).

Although chronic physical conditions are suggested to be an important predictor of mental health service use and 
psychopharmacological treatment (Crabb and Hunsley, 2006), the association with pain has received little attention. A recent study indicates an increased likelihood of receiving mental health care if a chronic pain condition is present (Braden et al., 2008). It is unclear whether the presence of pain in older adults is associated with a higher use of psychotropic medication either in depressed or in non-depressed older people. A pain condition and depression are both independently predictive for the use of benzodiazepines (Luijendijk et al., 2008), though the effect of the interaction is not clear.

The existing literature on pain and physical conditions in mental disorders in the elderly also lacks definitional consistency. Variables used to define pain and physical conditions vary widely and are sometimes poorly specified. This renders the research data difficult to compare.

The present study examines the relationship between pain and somatic conditions on the one hand, and depression on the other, in a large and representative sample of communitydwelling people aged 65 and over. Furthermore, the impact of pain and depression and their interaction on the use of mental health services and on the use of antidepressants and/ or benzodiazepines are explored.

\section{Materials and methods}

The data analyzed in this study is from the European Study on the Epidemiology of Mental Disorders (ESEMeD), which is part of the World Mental Health Surveys initiative of the World Health Organization. The target population was the noninstitutionalized older population of Belgium, France, Germany, Italy, the Netherlands and Spain. The study was cross-sectional in nature; a stratified, multistage, clustered area, probability sample was used. Out of the total of 21,425 adults (aged 18 or older) that formed the ESEMeD sampling frame, 4401 respondents aged 65+, were interviewed. 2716 (61.7\%) were aged $65-74$ and 1694 (38.3\%) were 75 or older. A more detailed description of the sampling frame and selection has been published elsewhere (Alonso et al., 2004).

Data were gathered by means of the Composite International Diagnostic Interview (CIDI 3.0; Kessler and Ustun, 2004; Haro et al., 2006). Major depressive episode (MDE) was diagnosed using the diagnostic criteria of DSM-IV (American Psychiatric Association, 1994). The presence of painful symptoms (PPS) was assessed through following questions: "Did you have back or neck problems or receive any treatment for them at any time during the past 12 months? Did you have frequent or severe headaches or receive any treatment for them at any time during the past 12 months? Did you have (any other) chronic pain or receive any treatment for it during the past 12 months?"

Participants were also asked if they had ever had any of the following chronic somatic conditions: arthritis or rheumatism, seasonal allergies, a stroke, a heart attack, heart disease, high blood pressure, asthma, tuberculosis, other chronic lung diseases, malaria or another parasitic disease, diabetes or high blood sugar, an ulcer in their stomach or intestine, a thyroid disease, a neurological problem, HIV, AIDS or cancer. A positive answer for any of these conditions was classified as a case of somatic condition. Respondents were asked about their use of antidepressants (including tricyclic and new generation antidepressants) and benzodiazepines in the last 12 months.
Table 1

Sociodemographic characteristics of the sample.

\begin{tabular}{lr}
\hline & $\%(\mathrm{SE})$ \\
\hline Mender & $40.6(.02)$ \\
Female & $59.4(.02)$ \\
Age & \\
65-74 years & $61.6(.02)$ \\
$75+$ years & $38.4(.02)$ \\
& \\
Married/cohabiting & \\
No & $34.6(.02)$ \\
Yes & $65.4(.02)$ \\
Urbanicity & \\
Rural & \\
Mid-sized urban & $36.3(.02)$ \\
Large urban & $37.1(.02)$ \\
& $26.6(.02)$ \\
Education & \\
$0-11$ years & \\
12 years & $52.0(.02)$ \\
13-15 years & $14.5(.01)$ \\
> =16 years & $26.3(.02)$ \\
\hline
\end{tabular}

Data were weighted to adjust for the multistage probability sampling. Population projection weights were used to restore the representativeness of the sample regarding age and gender distribution in each country. Prevalence estimates are provided and expressed in weighted percentages with standard error (SE/100). A logistic regression model was used to analyze factors associated with MDE, help-seeking and use of psychotropics (gender, age, somatic condition, pain, marital status, urbanicity, education). Statistical analyses were performed using STATA 9.0 statistical software (Statacorp, 2005).

\section{Results}

\subsection{Demographic characteristics}

The demographic characteristics of the sample are summarized in Table $1.59 \%$ of the respondents were female. One out of three lived alone. The majority of respondents were living in an urban or mid-size urban environment.

\subsection{The prevalence of PPS and MDE}

The 12-month prevalence of PPS was $31.8 \%$ (SE 0.02). PPS were more common in women than in men (35.9\% vs $25.9 \%$ ) and in those aged $75+$ compared to those aged 65-74 years (34.2\% vs 30.4\%). MDE was present in 128 respondents (2.6\%).

\subsection{The association between PPS and MDE}

The likelihood of PPS was greater in people with MDE than in those without (OR 2.06; 95\% CI 1.41-3.01). This reached statistical significance in females (OR 2.26; 95\% CI 1.48-3.43) but not in males (OR $1.31 ; 95 \% \mathrm{CI} 0.51-3.35)$. This increased risk of PPS in depressed respondents reached statistical significance in respondents with a somatic condition (OR 2.01; 95\% CI 1.333.02 ) but not in respondents without a somatic condition (OR $1.21 ; 95 \%$ Cl $0.41-3.51$ ). 
Table 2

Use of antidepressants and benzodiazepines (\%)-12 months.

\begin{tabular}{|c|c|c|c|c|c|c|c|c|c|}
\hline & \multirow[t]{2}{*}{ MDE } & \multirow[t]{2}{*}{ PPS } & \multicolumn{2}{|c|}{ Antidepressants } & & \multirow[t]{2}{*}{ MDE } & \multirow[t]{2}{*}{ PPS } & \multicolumn{2}{|c|}{ Benzodiazepines } \\
\hline & & & $\%$ & $(\mathrm{SE})$ & & & & $\%$ & (SE) \\
\hline (1) & - & - & 2.99 & $(0.61)$ & (1) & - & - & 11.28 & $(1.27)$ \\
\hline (2) & - & + & 8.32 & $(1.46)$ & (2) & - & + & 21.0 & $(2.85)$ \\
\hline (3) & + & - & 19.77 & $(6.28)$ & (3) & + & - & 49.09 & (6.19) \\
\hline (4) & + & + & 20.31 & $(5.50)$ & $(4)$ & + & + & 54.94 & (7.37) \\
\hline \multicolumn{5}{|c|}{$(1)=(2) F(1,142)=11.49$, prob $>F=0.0009$} & \multicolumn{5}{|c|}{$(1)=(2) F(1,142)=10.05$, prob $>F=0.0019$} \\
\hline \multicolumn{5}{|c|}{$(3)=(4) F(1,142)=0.00$, prob $>F=0.9499$} & \multicolumn{5}{|c|}{$(3)=(4) F(1,142)=0.21$, prob $>F=0.6460$} \\
\hline
\end{tabular}

Logistic regression analyses with MDE as the dependent variable and pain, gender, age, somatic condition, marital status, urbanicity and educational level as independent variables, were run to investigate further the relation between depression, pain and somatic conditions. Female gender $(\mathrm{OR}=3.75$; 95\% CI 1.97-7.14) and the presence of pain (OR $=2.56 ; 95 \% \mathrm{CI}$ 1.44-4.54) are predictors of the presence of MDE.

\subsection{Help-seeking}

Respondents without MDE but with PPS (4.42\%, SE $=1.20$ ) tended to seek more help for emotional problems, than respondents without MDE and without PPS $(1.99 \%, \mathrm{SE}=0.48)[(F$ $(1,142)=3.61$, prob $>F=0.0593$.

About one in three respondents with MDE sought help for emotional problems $[\mathrm{MDE}=29.3 \%(\mathrm{SE}=5.24)]$. There was no difference in rates of help seeking in respondents with MDE with PPS compared to respondents with MDE without PPS [MDE with PPS $=32.0 \% \quad(\mathrm{SE}=9.45)$; $\mathrm{MDE}$ without $\mathrm{PPS}=27.4 \%(\mathrm{SE}=6.15) ;(F(1,142)=0.16, \operatorname{prob}>F=.6914]$.

Logistic regression analyses indicated that respondents with MDE were significantly more likely to seek help for their emotional problems (OR $=7.56,95 \%$ CI 3.80-15.08). Respondents reporting PPS tended to seek more help ( $O R=1.72,95 \%$ CI 0.91-3.23) compared with those without PPS. Interaction effects of independent variables were not significant.

\subsection{Use of antidepressants}

In respondents without MDE, the presence of PPS resulted in a three-fold increase in the use of antidepressants (Table 2 ). About one out of five respondents with MDE reported the use of antidepressants in the last 12 months. No significant differences were found in respondents with or without PPS.

PPS, MDE and anxiety disorders were significant independent predictors of the use of antidepressants in the last 12 months (resp. OR $=2.35,95 \% \mathrm{CI} 1.40-3.97$; OR $=2.76,95 \%$ CI 1.34-5.67; OR =3.12, 95\% CI 1.27-7.68). Interaction effects of independent variables were not significant.

\subsection{Use of benzodiazepines}

In respondents without MDE, the presence of PPS resulted in a significant and substantial increase in the use of benzodiazepines (from 11 to $22 \%$ ) (Table 2). About one in two respondents with MDE reported the use of benzodiazepines in the last 12 months, and no significant differences were found in respondents with or without PPS.

PPS, MDE were significant predictors of the use of benzodiazepines in the last 12 months $(\mathrm{OR}=1.87,95 \% \mathrm{CI}$
1.25-2.81; OR $=5.11,95 \% \mathrm{CI} 3.05-8.54)$. Interaction effects of independent variables were not significant.

\section{Discussion}

In keeping with data from studies in adult populations and from studies in elderly, the likelihood of PPS was found to be twice as high in respondents with MDE (Demyttenaere et al., 2006; Ohayon and Schatzberg, 2003). The regression models further illustrate that depression and PPS are often comorbid and that they can be considered to be closely linked. The present study however illustrates that this increased prevalence of PPS is only found in respondents with a somatic condition. Indeed, this is in contrast with the (younger) adult population where depression resulted in an increased prevalence of PPS which was even more pronounced in respondents without a somatic condition than in respondents with a somatic condition (Demyttenaere et al., 2006). A possible explanation for this difference could be that, in the elderly, the increased prevalence of PPS is mainly due to a higher reporting of somatic condition-linked PPS due to depression while one could speculate that, in the general adult population, the increased prevalence of PPS could be more frequently due to somatization.

Gender seems to be a mediating factor in the relation between PPS and MDE. In congruence with other studies (Geerlings et al., 2002; Onder et al., 2005), we found a stronger association between pain and depression in women than in men. In pain literature, this gender effect has been explained by a difference in the extent of 'catastrophizing'. Women might magnify pain-related symptoms and are more pessimistic about pain-related outcomes (Keefe et al., 2000), which might lead to more psychological distress and consequently an enhanced risk of the comorbid presence of pain and depressive symptoms.

Only one out of three respondents with MDE sought help for emotional problems. This reflects the well documented under-recognition and undertreatment of psychiatric problems in this population (Tylee and Walters, 2007). MDE increases the help seeking for emotional problems (Braden et al., 2008). In contrast with the general adult population (where the presence of PPS decreased the likelihood of seeking help for emotional problems, Demyttenaere et al., 2006), older adults with PPS tend to seek more help (Braden et al., 2008). The presence of pain might be for present cohort of elderly a more decisive indication in the decision process of help-seeking than the experience of emotional problems. Future research is however needed to verify this hypothesis.

It is remarkable that in older respondents without MDE, the presence of PPS resulted in a 3-fold increase of the use of 
antidepressants. This could be partially due to the well documented efficacy of tricyclic antidepressants and serotonin and norepinephrine reuptake inhibitors in alleviating pain (Sindrup et al., 2005). It is also noteworthy that only about 1 in 5 respondents with MDE was using an antidepressant, illustrating that at least from a psychopharmacological point of view there is undertreatment (Lecrubier, 2007).

The overall use of benzodiazepines in our sample is rather high and is even higher in respondents with MDE where it reaches about $50 \%$ of the respondents (whereas only $20 \%$ were using an antidepressant). Again, in older respondents without MDE, the presence of PPS results in a 2-fold increase of the use of benzodiazepines. This could however be partially due to the known muscle orelaxant activity of benzodiazepines.

Despite the low rates of help-seeking for emotional problems in older adults with MDE, the rates of received psychopharmacological treatment are higher. This implies that older adults might have sought help and did receive some treatment, whereas the initial reasons for help-seeking were not attributed to emotional problems.

There are some limitations of our study which might hamper interpretation of results. First, the study was crosssectional, making it impossible to draw firm causal conclusions. Secondly, despite the large number of included respondents, the subgroup with caseness-level mental disorders is relatively small.

\section{Conclusions}

This large and representative community study in several European countries has demonstrated a strong and independent association between PPS and major depressive disorder. The overall use of mental health services is low. MDE and pain are independent factors increasing the rate of help seeking. The presence of PPS influences the use of psychotropic medication.

\section{Role of funding source}

The funding (see also Acknowledgements) did not interfere with the design, the analysis or the preparation of the manuscript.

\section{Conflict of interest}

The authors declare that they do not have a conflict of interest in submitting this manuscript.

\section{Acknowledgements}

This project was funded by the European Commission (Contract QLG5-1999-01042, SANCO 2004123), the Piedmont Region (Italy), Fondo de Investigación Sanitaria, Instituto de Salud Carlos III, Spain (FIS 00/0028), Ministerio de Ciencia y Tecnología, Spain (SAF 2000-158-CE), Departament de Salut, Generalitat de Catalunya, Spain, and other local agencies and by an unrestricted educational grant from GlaxoSmithKline. ESEMeD is carried out in conjunction with the World Health Organization World Mental Health (WMH) Survey Initiative.
We thank very much the WMH staff for assistance with instrumentation, fieldwork, and data analysis.

\section{References}

Alonso, J., Angermeyer, M.C., Bernert, S., et al., 2004. Sampling and methods of the European Study of the Epidemiology of Mental Disorders (ESEMeD) project. Acta Psychiatr. Scand., Suppl. 420, 8-20.

American Psychiatric Association, 1994. Diagnostic and Statistical Manual of Mental Disorders, 4th ed. APA. (DSM-IV).

Braden, J.B., Zhang, L., Fan, M., et al., 2008. Mental health service use by older adults: the role of chronic pain. Am. J. Geriatr. Psychiatry 16 (2), 156-167.

Chou, K.L., 2007. Reciprocal relationship between pain and depression in older adults: evidence from the English Longitudinal Study of Ageing. J. Affect. Disord. 102, 115-123.

Chou, K.L., Chi, I., 2005. Reciprocal relationship between pain and depression in elderly Chinese primary care patients. Int. J. Geriatr. Psychiatry 20, 945-952.

Crabb, R., Hunsley, J., 2006. Utilization of mental health care services among older adults with depression. J. Clin. Psychol. 62 (3), 299-312.

Demyttenaere, K., Bonnewyn, A., Bruffaerts, R., Brugha, T., De Graaf, R., Alonso, J., 2006. Comorbid painful physical symptoms and depression: prevalence, work loss, and help seeking. J. Affect. Disord. 92, 185-193.

Geerlings, S.W., Twisk, J.W.R., Beekman, A.T.F., Deeg, D.J.H., van Tilburg, W., 2002. Longitudinal relationship between pain and depression in older adults: sex, age and physical disability. Soc. Psychiatry Psychiatr. Epidemiol. 37, 23-30.

Haro, J.M., Arbabzadeh-Bouchez, S., Brugha, T.S., de Girolamo, G., Guyer, M.E., Jin, R., Lepine, J.P., Mazzi, F., Reneses, B., Vilagut, G., Sampson, N.A., Kessler, R.C., 2006. Concordance of the Composite International Diagnostic Interview Version 3.0 (CIDI 3.0) with standardized clinical assessments in the WHO World Mental Health Surveys. Int. J. Methods Psychiatr. Res. 15, 167-180.

Helme, R.D., Gibson, S.J., 2001. The epidemiology of pain in elderly people. Clin. Geriatr. Med. 17 (3), 417-431.

Hybels, C.F., Blazer, D.G., 2003. Epidemiology of late-life mental disorders. Clin. Geriatr. Med. 19 (4), 663-696.

Keefe, F.J., Lefebvre, J.C., Egert, J.R., Affleck, G., Sullivan, M.J., Caldwell, D.S., 2000. The relationship of gender to pain, pain behavior, and disability in osteoarthritis patients: the role of catastrophizing? Pain 87, 325-334.

Kessler, R.C., Ustun, T.B., 2004. The World Mental Health (WMH) Survey Initiative version of the World Health Organization (WHO) Composite International Diagnostic Interview (CIDI). Int. J. Methods Psychiatr. Res. 13, 93-121.

Lecrubier, Y., 2007. Widespread underrecognition and undertreatment of anxiety and mood disorders: results from 3 European studies. J. Clin. Psychiatry 68 (2), 36-41.

Luijendijk, H.J., Tiemeier, H., Hofman, A., Heeringa, J., Stricker, B.H.C., 2008. Determinants of chronic benzodiazepine use in the elderly: a longitudinal study. Br. J. Clin. Pharmacol. 65 (4), 593-599.

Meyer, T., Cooper, J., Raspe, H., 2007. Disabling low back pain and depressive symptoms in the community-dwelling elderly. Spine 32 (21), 2380-2386.

Ohayon, M.M., Schatzberg, A.F., 2003. Using chronic pain to predict depressive morbidity in the general population. Arch. Gen. Psychiatry 60 (1), 39-47.

Onder, G., Landi, F., Gambassi, G., 2005. Association between pain and depression among older adults in Europe: results from the Aged in Home Care (AdHOC) Project: a cross-sectional study. J. Clin. Psychiatry 66, 982-988.

Reid, M.C., Williams, C.S., Concato, J., Tinetti, M.E., Gill, T., 2003. Depressive symptoms as a risk factor for disabling back pain in community-dwelling older persons. J. Am. Geriatr. Soc. 51 (12), 1710-1717.

Sindrup, S.H., Otto, M., Finnerup, N.B., Jensen, T.S., 2005. Antidepressants in the treatment of neuropathic pain. Basic Clin. Pharmacol. Toxicol. 96 (6), 399-409.

Statacorp, 2005. Stata Statistical Software: Release 9. Statacorp LP, College Station, TX

Tylee, A., Walters, G., 2007. Underrecognition of anxiety and mood disorders in primary care: why does the problem exist and what can be done? J. Clin. Psychiatry 68 (2), 27-30.

Williamson, G.M., Schultz, R., 1992. Pain, activity restriction, and symptoms of depression among community-residing elderly adults. J. Gerontol. 47, 367-372. 